01,05,08, 18

\title{
Особенности поведения ионов $\mathrm{Mn}^{2+}$ в 3D-дираковском полуметалле $\alpha-\mathrm{Cd}_{3} \mathrm{As}_{2}$ по данным ЭПР
}

\author{
(С Ю.В. Горюнов ${ }^{1}$, А.Н. Натепров ${ }^{2}$ \\ ${ }^{1}$ ФИЦ „Казанский научный центр РАН“, \\ Казань, Россия \\ ${ }^{2}$ Институт прикладной фризики, \\ Кишинев, Молдавия \\ E-mail: gorjunov@kfti.knc.ru
}

Поступила в редакцию 12 сентября 2020 г.

В окочательной редакции 12 сентября 2020 г.

Принята к публикации 12 октября 2020 г.

\begin{abstract}
Методом ЭПР и электромагнитных измерений изучено поведение примеси марганца в 3D-дираковском полуметалле $\mathrm{Cd}_{3} \mathrm{As}_{2}$. Обнаружено, что в отличие от легирования европием, легирование марганцем, также как и железом, не приводит к изменению знака магнетосопротивления, которое при высоких концентрациях марганца почти полностью подавляется. При этом проявляется изменение характера влияния магнитного поля на контактный потенциал. Измеренные в ЭПР значения $g$-факторов совпадают с $g$-фактором свободного электрона при всех температурах, что с учетом флуктуационного поведения ширины линии ЭПР и редуцированного магнитного момента на $\mathrm{Mn}^{2+}$ указывает на образование короткоживущих связанных состояний иона $\mathrm{Mn}^{2+}$ и электронов проводимости - антиферромагнитных поляронов.
\end{abstract}

Ключевые слова: магнитный резонанс, дираковские полуметаллы, магнитные примеси.

DOI: 10.21883/FTT.2021.02.50463.196

\section{1. Введение}

В последнее десятилетие в связи с интенсивным развитием исследований перспективных материалов для изготовления различных функциональных устройств, в том числе для нужд развивающейся спинтроники, более пристальное внимание было обращено на арсениды металлов. В этот круг они попали, как вследствие обнаружения во многих из них зонных носителей тока с высокой подвижностью (InAs, GaAs) [1] или спиновой поляризацией (MnAs) [2], эффекта перпендикулярной магнитной анизотропии $\left(\mathrm{Mn}_{2} \mathrm{As}\right)$ [3], необычной связью энергии и импульса носителей тока $\left(\alpha-\mathrm{Cd}_{3} \mathrm{As}_{2}\right)[4,5]$. $\alpha-\mathrm{Cd}_{3} \mathrm{As}_{2}$ - это известный в прошлом полупроводник с узкой запрещенной зоной и высокой подвижностью электронов [6,7], а ныне известный, как 3D-дираковский топологический полуметалл [8]. Эти же материалы представляют фундаментальный интерес в плане изучения влияния примесей, тонких особенностей их внедрения в матрицу, на закон дисперсии носителей тока или спина и, наоборот, влияния закона дисперсии зонных носителей на взаимодействие примесей, в т. ч., немагнитных. Определенные достижения на этом пути получены, как в области получения химических соединений, обладающих такими свойствами, так и в области получения композитов [9] или наноструктур [10], изменяющих проводимость под влиянием магнитного поля, а также устройств, действующих на принципе переключении режима топологического полуметалла на обычный режим [11]. Тьюнинг топологических свойств посредством прецизионного допирования, как по пространственному расположению примеси в кристаллической решетке родительского соединения, так и по занимаемым карманам в энергетических зонах и количеству поставляемых или удаляемых электронов - перспективные пути решения этой задачи. Одним из интереснейших свойств топологических полуметаллов является защищенность электронных состояний в зоне проводимости от нарушений симметрии, из которого проистекают такие особенности в движении носителей тока, как эффекты слабой антилокализации или локализации в магнитном поле [12-15]. По всей видимости, с первым связана передача спиновых состояний на дальние расстояния, а со вторым влияние магнитного поля на химический потенциал носителей тока. В этой связи открывается обширное поле исследований влияния различных примесей, формирующих в топологическом материале рассеивающие центры различного характера $[14,15]$. Первые такие попытки $[16,17]$ была сделаны нами на примере введения магнитных примесей с нулевым орбитальным моментом в $3 \mathrm{D}$ топологический полуметалл $\alpha-\mathrm{Cd}_{3} \mathrm{As}_{2}$, представляемый, как объемный аналог графена $[4,5]$, и в котором дираковские узлы в зоне Бриллюэна дважды вырождены по спину. Магнитное поле и магнитные примеси, нарушая инверсионную симметрию пространства или обратимость времени, приводят к снятию этого вырождения и появлению в зоне Бриллюэна двух вейлевских узлов, разнесенных либо по энергии, либо по импульсу [18]. Линейный характер закона дисперсии зонных носителей может приводить к изменению РККИ взаимодействия [19-27], например, к появлению в нем 
дальнедействующей не осциллирующей части, связанной с топологической защищенностью зонных состояний. В то же время различия в рассеянии „родительских“ и донорных носителей тока на примесях определяют вид температурных и полевых зависимостей проводимости и магнитной восприимчивости легированного арсенида кадмия. Исследования легированного марганцем $\alpha-\mathrm{Cd}_{3} \mathrm{As}_{2}$ проводились в 80-е годы прошлого столетия $[6,7]$ и уже тогда отмечался линейный характер закона дисперсии и высокая подвижность зонных носителей, обсуждалась роль РККИ-взаимодействия между ионами $\mathrm{Mn}^{2+}$. Однако, несмотря на наличие большого интереса к этому соединению и в прошлом и в настоящем, исследований поведения примеси марганца в $\alpha-\mathrm{Cd}_{3} \mathrm{As}_{2}$ методом ЭПР не проводились. Ряд новых полученных в этой области результатов мы излагаем в настоящей статье.

\section{2. Эксперимент}

Слитки состава $\left(\mathrm{Cd}_{1-x} \mathrm{Mn}_{x}\right)_{3} \mathrm{As}_{2}$, где $x$ равен 0.05 и 0.1 были синтезированы при $1023 \mathrm{~K}$ путем как прямой реакции составляющих элементов: $\mathrm{Cd}$ чистотой $99.999 \%$, As - 99.9999\%, Mn 99.99\%, так и через сплавы $\mathrm{Cd}-\mathrm{Mn}$, в стеклографитовых тиглях помещенных в вакуумированные отпаянные кварцевые ампулы. Кристаллическая структура и стехиометрия проверялась с помощью рентгеновской порошковой дифракции (PXRD) и рентгеновского флуоресцентного анализа (EDX). Измерения полевых и температурных зависимостей электросопротивления производились стандартным четырехзондовым методом в температурном интервале $10-300 \mathrm{~K}$ и магнитных полях до 2Т. Измерения ЭПР и нерезонансного поглощения СВЧ излучения проводились на стандартном ЭПР спектрометре „Брукер“ в Х-диапазоне $(9.22 \mathrm{GHz})$ и при температурах 8-300 K. Для измерений $g$-фактора использовалось добавление в порошок образца маркера - ДФПГ, имеющего очень узкую резонансную линию и точно известное значение $g$-фактора $2.0036 \pm 0.0002[28]$.

\section{3. Результаты эксперимента}

\section{1. Структура и состав образцов}

Полученные данные PXRD подтверждают идентичность синтезированных чистых и легированных соединений низкотемпературной тетрагональной модификации $\alpha-\mathrm{Cd}_{3} \mathrm{As}_{2} \quad$ [5] с пространственной группой симметрии $\mathrm{I}_{1} \mathrm{~cd}$ и параметрами решетки $a=b=12.643 \AA, c=25.431 \AA$, для $x=0.05$ и $a=b$ $=12.591 \AA, \quad c=25.327 \AA$ для $x=0.1$. Параметры кристаллической решетки чистого $\alpha-\mathrm{Cd}_{3} \mathrm{As}_{2}$, полученного тем же способом, составляли $a=b=12.6539 \AA$, $c=25.4586 \AA$. Экспериментальные и расчетные данные по PXRD, как и в $[16,17]$ практически совпадают и показывают ожидаемое из сравнения размеров ионов $\mathrm{Cd}^{2+}$ и $\mathrm{Mn}^{2+}$ уменьшение параметров решетки в сравнении с чистым материалом. Каких-либо других дополнительных фаз и соединений обнаружено не было. Однако, данные EDX, подтверждая сохранение стартовой стехиометрии, указывают на некоторый дефицит по мышьяку, связанный с небольшим испарением мышьяка из стеклографитового тигля и его конденсацией на стенках ампулы при остывании слитка, а оценки восприимчивости указывают на следы ферромагнитной фазы MnAs $\left(T_{c}=320 \mathrm{~K}\right) \quad[2,6,7,9,29]$. Оценка из данных по температурной зависимости магнитной восприимчивости образца с $x=0.1$ количества марганца вовлеченного в MnAs дает величину не более $7.3 \%$, т.е. без учета возникающего небольшого дефицита по мышьяку вместо $x=0.1$ следует иметь ввиду $x=0.093$. Таким образом, при оценке влияния концентрации примеси это требует проведения лишь небольшой корректировки конечного состава слитка и будет обсуждаться в дальнейшем. Также следует иметь ввиду, что примесь марганца, также как и примесь европия [16], в кристаллической решетке $\alpha-\mathrm{Cd}_{3} \mathrm{As}_{2}$, имеющей тип решетки антифлюорита, может располагаться на месте ионов кадмия (позиции замещения) и в междоузлиях (позиции внедрения) 0.1 at.\% Сd при 298 К [30,31]).

\section{2. Электросопротивление}

При комнатной температуре удельное электросопротивление $\left(\mathrm{Cd}_{1-x} \mathrm{Mn}_{x}\right)_{3} \mathrm{As}_{2}$ оценивается: для $x=0.05 \rho=0.17 \pm 0.05 \mathrm{~m} \Omega \cdot \mathrm{cm}$, для $x=0.1$ $\rho=0.3 \pm 0.05 \mathrm{~m} \Omega \cdot \mathrm{cm} . \quad$ В обоих случаях электросопротивление имеет металлический характер температурной зависимости (см. рис. 1), как и при легировании европием [16]. Однако, в отличие от легирования европием, линейная температурная зависимость при абсолютном нуле попадает в область

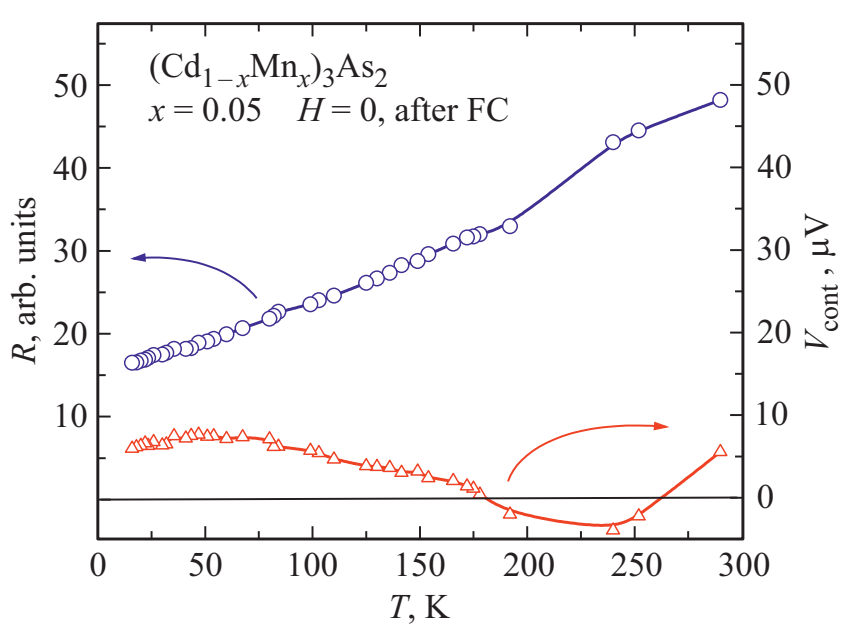

Рис. 1. Температурная зависимость электросопротивления и контактной разницы потенциалов в электротранспортных измерениях $\left(\mathrm{Cd}_{1-x} \mathrm{Mn}_{x}\right)_{3} \mathrm{As}_{2}$. 


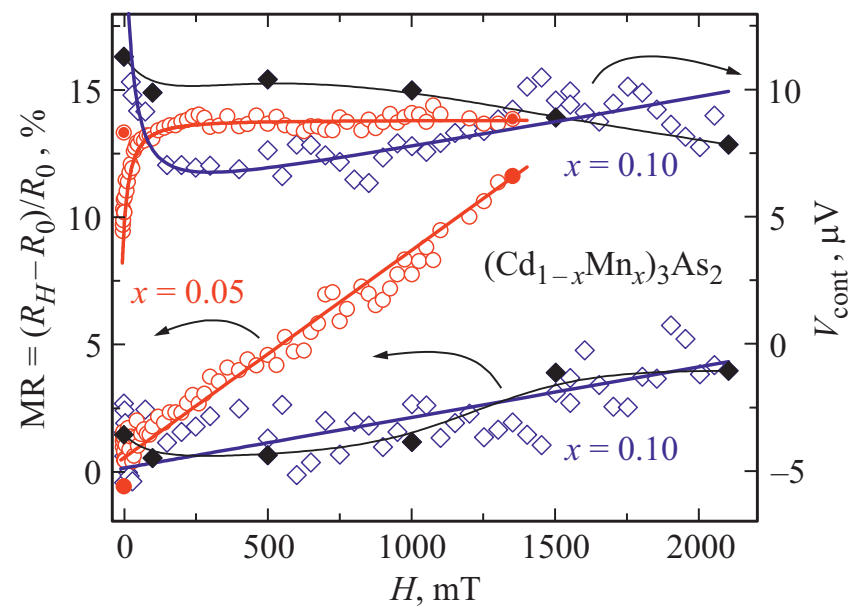

Рис. 2. Полевые зависимости магнетосопротивления и контактного потенциала. Кружки - образец $\left(\mathrm{Cd}_{1-x} \mathrm{Mn}_{x}\right)_{3} \mathrm{As}_{2} \mathrm{c}$ $x=0.05$, ромбы - с $x=0.1$. Полные значки - зависимости сняты на убывающем поле.

положительных сопротивлений, имеет аномалию вблизи 200-250 K, термоЭДС (или контактный потенциал) в этом диапазоне температур меняет знак. Сравнение магнетосопротивлений (MR) чистого и легированных марганцем образцов показали существенное влияние степени легирования на полевые зависимости MR. Для чистого образца и образцов, легированных марганцем, при комнатной температуре наблюдались следующие положительные $\mathrm{MR}=\left(R_{(H=1.6 \mathrm{~T})}-R_{(H=0)}\right) / R_{(H=0)}$ :

$$
\begin{gathered}
\operatorname{MR}\left(x_{\mathrm{Mn}}=0\right)=0.468 ; \quad \operatorname{MR}\left(x_{\mathrm{Mn}} \sim 0.05\right)=0.137 ; \\
\operatorname{MR}\left(x_{\mathrm{Mn}} \sim 0.1\right)=0.040 .
\end{gathered}
$$

При понижении температуры до $29 \mathrm{~K} \operatorname{MR}\left(x_{\mathrm{Mn}} \sim 0.05\right)$ возрастало в $\sim 2.2$ раза. (Для примеси железа, соответственно, $\mathrm{MR} \sim 0.2$ и возрастало в $\sim 5$ раз [17]). В случае легирования европием [16] наблюдалось отрицательное MR $=-0.213$.

Таким образом, полевые зависимости сопротивления образцов $\left.\mathrm{Cd}_{1-x} \mathrm{Mn}_{x}\right)_{3} \mathrm{As}_{2}$ были слабые и имели близкий к линейному характер (см. рис. 2). В то же время для обоих образцов наблюдалась полевая зависимость контактной разности потенциалов, выражающаяся в следующем. В малых полях происходило заметное изменение контактного потенциала (увеличение для $x=0.05$ и уменьшение для $x=0.1)$ при увеличении магнитного поля от небольших отрицательных значений до $\sim 50 \mathrm{mT}$. При дальнейшем увеличении магнитного поля в обоих случаях контактный потенциал оставался на уровне $8-9 \mu \mathrm{V}$ в более высоких полях и при возвращении в нулевое поле. При температуре $29 \mathrm{~K}$ он был вдвое меньше.

\section{3. Эектронный парамагнитный резонанс}

Для всех порошковых образцов во всем изученном температурном диапазоне на первый взгляд наблюдалась интенсивная одиночная линия ЭПР практически идеальной лоренцевой формы с небольшими дайсоновскими искажениями (см. рис. 3). Качественные спектры позволили провести тщательное моделирование спектров, которое показало следующее. Небольшие дайсоновские искажения описываются примесью дисперсии к линии поглощения, которая при комнатной температуре составляла $\sim 4 \%$ для $x=0.1$ и $\sim 8 \%$ для $x=0.05$ ). Лоренцева форма резонансной линии указывает на сильный эффект обменного сужения [32-34]. При комнатной температуре пиковая ширина линии и уточненный при моделировании спектра $g$-фактор составляли: для $x=0.05 \Delta H_{p p}=15.5 \mathrm{mT}, g=2.0024 \pm 0.0006$, для $x=0.1 \Delta H_{p p}=13.0 \mathrm{mT}, g=2.0021 \pm 0.0006$. Т. е. $g$-фактор всех образцов в пределах точности измерений совпадал с $g$-фактором свободного электрона $g=2.0023$. При понижении температуры происходило небольшое (мнимое) смещение резонансной линии в сторону высоких полей (более сильное $\sim 4 \mathrm{mT}$ на уровне $H_{\text {res }} \sim 330 \mathrm{mT}$ для менее легированного образца с $x=0.05)$ и ее уширение до $\Delta H_{p p} \sim 60 \mathrm{mT}$, причем более быстрое для образца с $x=0.1$, изначально имеющего более узкую линию (см. рис. 4). Однако, тщательное моделирование спектра вместе с резонансной линией маркера показало, что в спектре присутствует слабая более широкая, чем основная, линия, учет которой вместе с дайсоновскими искажениями позволил полностью (см. рис. 3) описать все экспериментальные спектры. При этом выяснилось, что с понижением температуры в резонансных линиях слегка увеличивается примесь дисперсии, которая также различается для различных образцов, а действительный сдвиг резонансного поля отсутствует во всем исследованном диапазоне температур. Вклад дополнительной резонансной линии так-

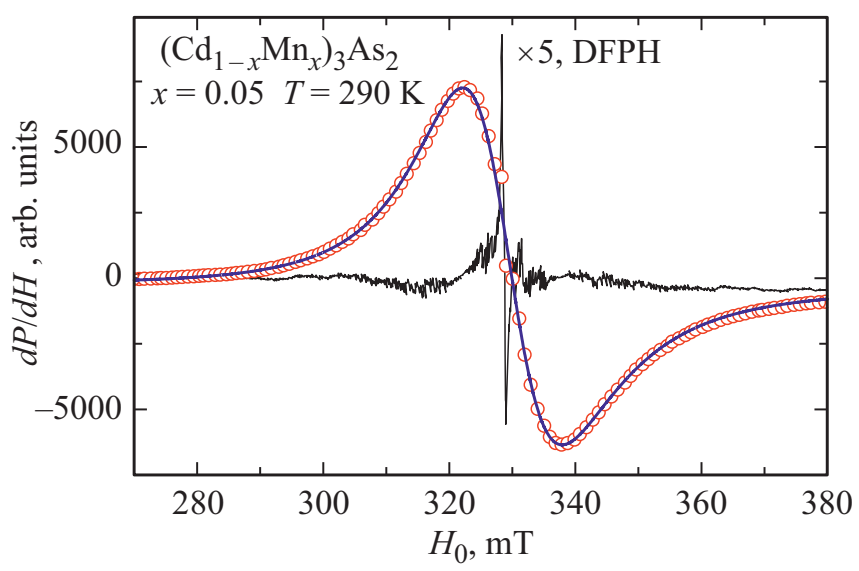

Рис. 3. Моделирование экспериментальных спектров. Кружки - экспериментальные точки, сплошная линия расчет спектра для $x=0.05$, состоящего из двух линий с $g_{1}=2.0024$, $\Delta H_{p p}^{(1)}=15.5 \mathrm{mT} ; g_{2}=1.954, \Delta H_{p p}^{(2)}=21.4 \mathrm{mT}$, интенсивностями $I_{2}: I_{1}=1: 15$. Спектр с узкой линией ДФПГ - разница между экспериментом и расчетом. $H_{\mathrm{DFPH}}=328.7 \mathrm{mT}$ $(g=2.0036)$. 


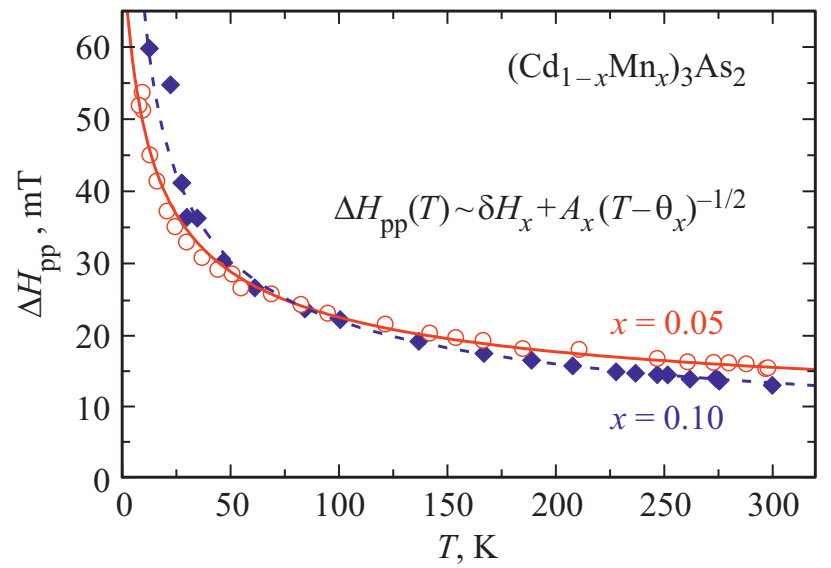

Рис. 4. Температурная зависимость ширины линии ЭПР на ионах $\mathrm{Mn}^{2+}$ в $\left(\mathrm{Cd}_{1-x} \mathrm{Mn}_{x}\right)_{3} \mathrm{As}_{2}$. Пустые кружки $-x=0.05$, полные ромбы $-x=0.1$.

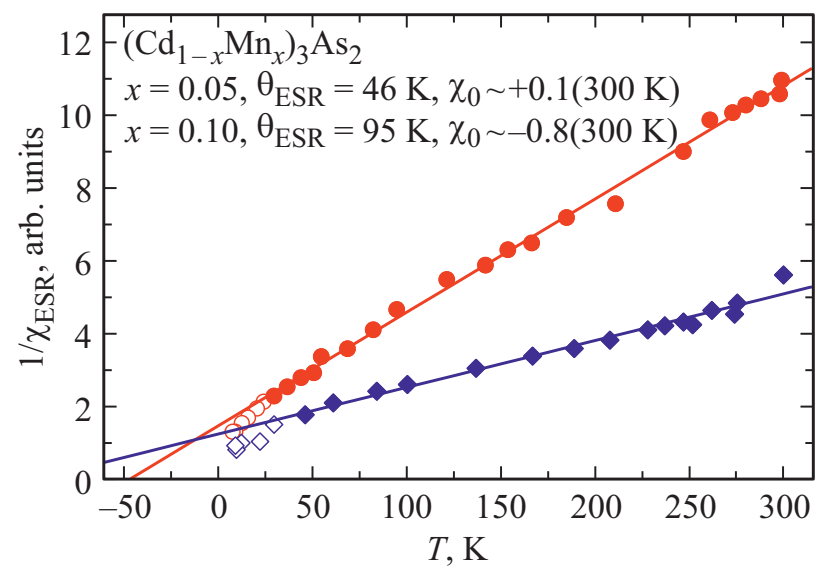

Рис. 5. Температурные зависимости обратной интенсивности спектров для образцов с $x=0.05$ (кружки), $x=0.1$ (ромбы) с учетом не зависящего от температуры вклада в интенсивность линии ЭПР (указаны на рисунке). Температуры Кюри-Вейса образцов близки к известным из работ $[6,42]$, однако, не зависящие от температуры вклады получаются различными. Это может быть объяснено своеобразной конкуренцией диамагнитного (Ланжевен, Ландау) и парамагнитного (Паули) вкладов в процессе заполнения зоны проводимости по мере увеличения концентрации допирующей примеси $\mathrm{Mn}$ (донорных электронов) и вклада в восприимчивость образца ферромагнитных кластеров MnAs.

же различается для различных образцов. Для $x=0.05$ соотношение интенсивностей дополнительной линии к основной составляет $1: 15$, а для $x=0.1-1: 20$.

Положение и ширина дополнительной линии относительно $g=2.0023$ также различно для различных образцов. Для образца с $x=0.05$ эта линия имеет $g=1.954 \pm 0.005 \Delta H_{p p}=21.4 \mathrm{mT}$, а для $x=0.1$ эта линия имеет $g=2.044 \pm 0.005 \Delta H_{p p}=21.1 \mathrm{mT}$. С понижением температуры дополнительная линия также, как и основная линия ЭПР, уширяется и достоверно выделить ее не удается, т. к. спектр хорошо описывается одиночной лоренцевой линией. На данный момент дать однозначный ответ на вопрос о природе дополнительной линии в определенной степени затруднительно. Однако, учитывая, что в образце имеются следы ферромагнитной фазы MnAs, мы можем отнести данный сигнал к ней либо к основной фазе. Согласно [9,29,35-41] данное соединение обладает достаточно большой спонтанной намагниченностью и гексагональной структурой, дающую, как правило, большую магнитную анизотропию. Собственно, это соединение и известно, как соединение с очень большой степенью поляризации зоны проводимости. Все это означает, что в случае порошкового образца наблюдение сигнала ферромагнитного резонанса от MnAs невозможно. Тем более, невозможно наблюдение сигнала низкой интенсивности. Таким образом, с большой долей вероятности мы можем отнести дополнительный сигнал к основной фазе $\alpha-\mathrm{Cd}_{3} \mathrm{As}_{2}$. По аналогии с ЭПР на ионах $\mathrm{Eu}^{2+}$ в данном соединении, эту линию можно отнести к ионам $\mathrm{Mn}^{2+}$, разместившимся в позициях внедрения. Однозначно признать это мешает то обстоятельство, что кристаллохимический радиус иона $\mathrm{Mn}^{2+}$ на $9 \%$ меньше такового для иона $\mathrm{Cd}^{2+}$, и ион $\mathrm{Mn}^{2+}$ не испытывает таких различий степеней химического сжатия в различных позициях, как это имеет место для иона $\mathrm{Eu}^{2+}$. Однако, возможен сдвиг линий ЭПР ионов $\mathrm{Mn}^{2+}$, находящихся в различных кристаллографических позициях, вследствие различия в этих позициях плотностей $s$-электронов, с которыми связан электронный сдвиг Найта.

Температурные зависимости обратной интенсивности, которая пропорциональна селективной магнитной восприимчивости ионов в данной матрице, имели нелинейный характер. Данная нелинейность исчезала при учете независящего от температуры вклада (см. рис. 5). При этом парамагнитные температуры Кюри попадали в диапазон значений, соответствующий, ранее полученным в $[6,7,42]$ для соответствующих концентраций. Однако, независящие от температуры вклады оказались несколько отличными от этих данных. Это может быть объяснено наличием двух конкурирующих вкладов: диамагнитного от матрицы $\mathrm{Cd}_{3} \mathrm{As}_{2}$ и парамагнитного от поляризующего эффекта ферромагнитных кластеров MnAs в относительно высоких резонансных полях.

\section{4. Обсуждение}

Ион $\mathrm{Mn}^{2+}$ имеет электронную конфигурацию незаполненной оболочки $-3 d^{5}$. Для основного состояния иона марганца $(2+)$ его квантовые числа $S=5 / 2, L=0$, $J=5 / 2$. Тонкая структура спектра ЭПР, обусловленная кристаллическим полем, для такого набора квантовых чисел предполагает наличие пяти резонансных линий. Вследствие взаимодействия магнитных моментов неспаренных электронов с магнитным моментом ядра образуется сверхтонкая структура спектра. Спин ядра марганца $I=5 / 2$. Т. е. под действием внешнего магнитного поля 
магнитный момент ядра принимает $2 I+1=6$ различных направлений, вследствие чего каждый электронный зеемановский подуровень расщепляется на шесть подуровней сверхтонкой структуры, в результате этого спектр оказывается состоящим из 30 линий [33,34]. Если учесть взаимодействие с ядрами лигандов, имеющих ненулевой спин, то в спектре ЭПР проявляется еще и аналогичная суперсверхтонкая структура, обусловленная как наличием спина ядер лигандов, так и конфигурацией их расположения [43]. Это как раз случай $\alpha-\mathrm{Cd}_{3} \mathrm{As}_{2}$, где ядра $\mathrm{As}$ и два естественных изотопа $\mathrm{Cd}$ имеют ненулевые спины ядер. Таким образом, спектр ЭПР иона $\mathrm{Mn}^{2+}$ может состоять из более сотни линий поглощения ЭПР. Учитывая, что кристаллическое поле в структуре типа антифлюорита мало $\left(\sim 10^{-3}\right.$ от резонансного поля [44]), то размах сверхтонкой структуры может быть не более $50 \mathrm{mT}$. Соответственно, среднее расстояние между резонансными линиями (и энергетическими состояниями) около $0.5 \mathrm{mT}$ и в случае обменного сужения весь этот массив линий всегда образует единственную резонансную линию лоренцевой формы. В [34] указывается, что при обменном сужении все-таки крылья резонансной линии имеют остатки гауссовой формы. Следует заметить, что в нашем случае этого не наблюдается, очевидно, из-за большой ширины начальной структуры спектра и большим числом включенных в нее межконфигурационных переходов.

Увеличение доли примеси дисперсии в спектральной линии и относительное изменение амплитуд низкополевого и высокополевого пика производной линии поглощения по полю с понижением температуры может объясняться двояко: увеличением скорости спиновой диффузии и уменьшением электросопротивления образца. Уменьшение электросопротивления закономерно приводит к уменьшению скин-слоя до величины сравнимой с размером частиц порошка. Версия, что изменение доли дисперсии обусловлено изменением сопротивления образцов, хорошо согласуется как с температурным поведением сопротивления образцов, так и с измеренными величинами их удельных сопротивлений: образец с более высокой концентрацией примеси марганца имеет большее сопротивление, больший скин-слой на резонансной частоте и меньшую долю дисперсии в резонансном поглощении. При таком дайсоновском [45] искажении резонансной линии точка на ней, соответствующая резонансному полю, смещается ближе к низкополевому пику. Поэтому при расчете резонансного поля по середине между пиками получаем завышенное резонансное поле вследствие смещения высокополевого пика, вызванного уширением линии и увеличением примеси дисперсии. Т. е. симуляция спектров дает более правильный результат: полное отсутствие температурной зависимости $g$-фактора. Таким образом, измеренные в ЭПР значения $g$-факторов показывают их полное совпадение с $g$-фактором свободного электрона, независимость от температуры и, следовательно, полную компенсацию всех обменных взаимодействий и кристаллических полей, приводящих к изменению величины $g$-фактора чисто спинового состояния иона $\mathrm{Mn}^{2+}$.

Вместе с тем наблюдается сильная температурная зависимость ширины линий. Для обоих образцов температурные зависимости хорошо описываются выражением типа $\Delta H(T)=\Delta H_{0}+\Delta H_{t} \cdot\left(T-T_{c}\right)^{-1 / 2}$, следующего для магнитных флуктуаций из теории Ландау фазовых переходов второго рода [46]. Здесь, $\Delta H_{0}-$ независящая от температуры обменная ширина линии, связанная с частотой обмена между ионами $\mathrm{Mn}^{2+} ; \Delta H_{t}$ - температурный коэффициент, $T_{c}$ - температура магнитного фазового перехода.

Из факта, что величина $g$-фактора чисто спинового состояния $\mathrm{Mn}^{2+}$ в точности равна $g$-фактору свободного электрона, следует, что магнитный момент иона $\mathrm{Mn}^{2+}$ должен был бы соответствовать величине $\sim 5.9 \mu$ В. Однако, ранее выполненные измерения $[6,7,42]$ магнитной восприимчивости указывают на величину $4.4 \mu \mathrm{B}$. Принимая во внимание флуктуационное поведение ширины линии ЭПР мы можем прийти к выводу об образовании при низких температурах коротко живущих связанных состояний иона $\mathrm{Mn}^{2+}$ и электронов проводимости антиферромагнитных поляронов. Антиферромагнитные флуктуации не приводят к изменению резонансного поля, но дают флуктуационный вклад в ширину линии в соответствии с выражением для магнитных флуктуаций в теории фазовых переходов Ландау. Температурное поведение ширины линии описывается двумя вкладами. Первый вклад $\Delta H_{0}$ - это по умолчанию независящий от температуры вклад, обусловленный обменными взаимодействиями, приводящими к сужению резонансной линии. Этот вклад в ширину линии связан с обменом между спиновыми состояниями и сужение резонансной линии тем больше, чем выше обменная частота (см. [32-34]). Поскольку обменная частота увеличивается с увеличением концентрации взаимодействующих ионов, то обменно-суженная резонансная линия в отсутствие других вкладов имеет меньшую ширину для большей концентрации магнитных примесей. Второй вклад, зависящий от температуры, связан с магнитными флуктуациями. В случае ферромагнитных флуктуаций он дает вклад и в значение резонансного поля. Образование короткоживущего антиферромагнитного полярона на ионе $\mathrm{Mn}^{2+}$ является механизмом, объясняющем наблюдаемое понижение магнитного момента на ионе $\mathrm{Mn}^{2+}$. Взаимодействие со свободным электроном, имеющим тот же $g$-фактор не приводит к изменению эффективного g-фактора полярона [47], но уменьшает величину его эффективного магнитного момента, наблюдаемую в измерениях статической магнитной восприимчивости. Возможно, что небольшое (в пределах ошибки) увеличение $g$-фактора для образца с меньшей концентрации $\mathrm{Mn}^{2+}$ может быть вызвано взаимодействием с электронами проводимости, имеющими аномально 
высокие $g$-факторы, которые свидетельствуют о частичном сохранении топологического состояния для этой концентрации примеси.

Значения $g$-фактора дополнительной линии, которую мы отнесли к сигналу от ионов $\mathrm{Mn}^{2+}$, находящихся в тетраэдрических вакансиях для различных образцов, оказались различающимися на два порядка больше, чем различия $g$-факторов от основной линии: $g=1.954 \pm 0.005(x \sim 0.05)$ и $g=2.044 \pm 0.005$ $(x \sim 0.1)$ при практически одинаковой пиковой ширине линии $\sim 21 \mathrm{mT}$. Объяснение этому достаточно простое. Если допустить, что практически все донорные электроны поступают в зону проводимости из $s$-оболочки марганца, то на данных ионах плотность $s$-электронов, дающих основной вклад в электронный сдвиг Найта, практически нулевая. Это распределение электронной плотности определяется электронной структурой соединения, его симметрией, которую в этом плане двухвалентный ион $\mathrm{Mn}^{2+}$, замещая $\mathrm{Cd}^{2+}$, не нарушает. Этого нельзя сказать о таких же ионах марганца, расположенных в позициях внедрения, в тетраэдрических вакансиях. В этих позициях плотность $s$-электронов может быть не нулевая и, как следствие, иметь поляризацию, давать электронный сдвиг Найта и вклад в ширину линии. Очевидно, что за счет $s-d$ связи это будут те же вклады (но сильнее) в ширину резонансной линии, как и для ионов в позициях замещения. Электронный сдвиг Найта будет иметь тот же знак, как и знак поляризации плотности $s$-электронов на ионе $\mathrm{Mn}^{2+}$. Если это будут электроны, поляризованные против внешнего магнитного поля, то мы получим отрицательный сдвиг $g$-фактора относительно такового для свободного электрона, и наоборот. Примерно такую картину мы наблюдаем для наших образцов: для образца с меньшей концентрацией марганца - отрицательный сдвиг $g$-фактора -0.04, для образца с большей концентрацией марганца положительный сдвиг $\sim+0.04$, по порядку величины соответствующие магнитной восприимчивости электронных систем, обуславливающих эти сдвиги. С увеличением концентрации примеси уменьшается относительное содержание второй фазы с $1: 15(x=0.05)$ до $1: 20$ $(x=0.1)$, что свидетельствует об очевидном ограничении на расположение ионов $\mathrm{Mn}^{2+}$ в позициях внедрения и первоначально предпочтительном их размещении в этих позициях. Данные результаты закономерно выводят на объяснение полученных температурных и полевых зависимостей электросопротивления данных образцов. В образце с малой концентрацией марганца мы наблюдаем значительное положительное магнетосопротивление (см. рис. 2), свидетельствующее о сохранении режима Дираковского топологического полуметалла в данном образце и, соответственно, наличии эффекта слабой антилокализации и о диамагнитном отклике электронов зоны проводимости матрицы. Вместе с тем, в образце, согласно данным о магнитной восприимчивости и данным [6-9,48,49], присутствуют ферромагнитные кластеры MnAs, которые при температурах ниже $T_{f}=241 \mathrm{~K}$, переходят в состояние подобное спиновому стеклу. Этот переход проявляется в аномалии на температурной зависимости электросопротивления данного образца (см. рис. 1). Наличие ферромагнитных кластеров, инжектирующих [50] в зону проводимости поляризованные $s$-электроны, изменяет распределение электронов между поляризациями вблизи кластеров, но не меняет положение уровня Ферми и величину контактного (химического) потенциала в среднем по образцу в отсутствие его макроскопической намагниченности. MnAs известен в качестве термомагнитного [51] полупроводникового материала, который, имея сильно поляризованную зону проводимости, изменяет положение уровня Ферми, химический и контактный потенциал в зависимости от своей намагниченности. Таким образом, в случае низкой проводимости MnAs в композите $\mathrm{Cd}_{3} \mathrm{As}_{2}-\mathrm{MnAs}$, сопротивление образца будет определяться свойствами матрицы и распределением ее электронов по подзонам с различной поляризацией. Если матрица сохраняет свойства топологического полуметалла и рассеяние назад инжектируемых из нормального металла электронов подавлено, то при отсутствии макроскопической намагниченности кластеров вдоль границы металл-дираковский полуметалл концентрации электронов любых поляризаций с обеих сторон от границы выравниваются и контактный потенциал устанавливается на некотором минимальном уровне. С появлением в дираковском полуметалле макроскопической поляризации электронов проводимости, обусловленной кластерами MnAs, контактный потенциал возрастает до выравнивания уровней Ферми по обе стороны границы. Поскольку в отсутствие режима дираковского полуметалла передачи поляризаций электронов проводимости, вызванными неупорядоченными ферромагнитными кластерами, и их усреднения вдоль границы раздела не происходит, то контактный потенциал устанавливается на некотором максимальном уровне. Упорядочение ферромагнитных кластеров внешним магнитным полем приводит к спаданию контактного потенциала. Поэтому в электротранспортных измерениях наблюдается гистерезис, связанный гистерезисом намагниченности ферромагнитных кластеров MnAs [40,41].

И в заключение несколько замечаний по результатам других исследований системы $\left(\mathrm{Cd}_{1-x} \mathrm{Mn}_{x}\right){ }_{3} \mathrm{As}_{2}$ и о механизме образования кластеров MnAs в этой системе. В настоящей работе, а также в $[42,48]$, замечено, что с увеличением концентрации примеси марганца образование фазы MnAs, имеющей температуру плавления $T=1208 \mathrm{~K}$ несколько выше температуры плавления $\mathrm{Cd}_{3} \mathrm{As}_{2} 994 \mathrm{~K}$, начинается при концентрациях $x>2 \%$ Мn и при $x \sim 0.1$ уже $7.3 \%$ всего марганца уходит в эту фазу. Несмотря на то, что выращивание кристаллов $\mathrm{Cd}_{3} \mathrm{As}_{2}$, легированных $\mathrm{Mn}$, в работе [48] производилось методом молекулярно лучевой эпитаксии и при низких температурах $(453 \mathrm{~K})$, при концентрациях $x>0.02$ происходило образование второй фазы, т. е. нарушалась 
гомогенность образца. Очевидной причиной этому является отсутствие гибридизации валентных орбиталей MnAs c валентными орбиталями $\mathrm{Cd}_{3} \mathrm{As}_{2}$, аналогично тому, как это имеет место в случае системы $\mathrm{Fe}-\mathrm{Pb}$, компоненты которой не смешиваются даже в жидком состоянии.

B композитах [9] содержание компонентов MnAs $\left(47 \mathrm{~mol} \%\right.$ ) и $\mathrm{Cd}_{3} \mathrm{As}_{2}$ в их эвтектике примерно равное, но в указанной работе не называется концентрация растворенного в $\mathrm{Cd}_{3} \mathrm{As}_{2}$ марганца. Между тем, согласно [52,53], соединение $\mathrm{Mn}_{3} \mathrm{As}_{2}$ не обладает той же структурой, что $\alpha-\mathrm{Cd}_{3} \mathrm{As}_{2}$. Это означает, что нет непрерывного перехода между $\alpha-\mathrm{Cd}_{3} \mathrm{As}_{2}$ и $\mathrm{Mn}_{3} \mathrm{As}_{2}$ и существует предел растворимости марганца в арсениде кадмия. Однако, как можно сделать вывод из данных [42] и [48], количество растворенного в $\mathrm{Cd}_{3} \mathrm{As}_{2}$ марганца находится в определенном равновесии с числом выпадающих в нем жидких кластеров MnAs. Согласно работе [9], при высоком содержании марганца фаза MnAs образует в арсениде кадмия кластеры размером $30-50 \mathrm{~nm}$. Возможно, что размер кластеров связан с зависимостью температуры плавления наночастиц от их размера [54]. T. е. мелкие частицы MnAs, имеющие температуру плавления ниже температуры плавления арсенида кадмия при затвердевании эвтектики не образуются. Вследствие существования зависимости температуры плавления наночастиц от их размера и относительной близости температуры синтеза (1023 K) к температурам плавления массивных $\mathrm{Cd}_{3} \mathrm{As}_{2}(994 \mathrm{~K}), \mathrm{MnAs}(1208 \mathrm{~K})$ и их эвтектики, в жидкой фазе устанавливается равновесие между количеством растворенного в $\mathrm{Cd}_{3} \mathrm{As}_{2}$ марганца и каплями MnAs, размер и количество которых также определяется конкуренцией поверхностной энергии и теплотой плавления наночастицы [52]. Такой механизм, по сути, подтверждается результатами работы [9]. Таким образом, на основании наших наблюдений и данных работ $[9,42,48]$, мы можем сделать вывод, что при концентрациях растворенного марганца $x>0.02$, в $\mathrm{Cd}_{3} \mathrm{As}_{2}$ образуются капли MnAs, в которые при $x \sim 0.10$ переходит около $7.3 \%$ содержащегося в образце марганца. При линейной интерполяции для нашего образца с $x=0.05$ получаем, что в этом образце, сохраняющим признаки дираковского полуметалла и содержащего около $2.5 \%$ марганца в MnAs-фазе, концентрация $\mathrm{Mn}^{2+}$ в $\alpha-\mathrm{Cd}_{3} \mathrm{As}_{2}$ составляет $x \sim 0.049$, а размеры областей, свободных от MnAs, составляют $\sim 500 \mathrm{~nm}$.

В работе Маренкина [49], выполненной на пленочных образцах арсенида кадмия, легированных ионами $\mathrm{Mn}^{2+}$, указывается на критическую концентрацию ионов марганца $x_{c} \sim 0.023$ (в наших обозначения), при которой происходит переход от режима слабой антилокализации (САЛ) с положительным MR к режиму слабой локализации (СЛ) с отрицательным MR и разрушении топологического состояния при увеличении концентрации магнитной примеси. В наших экспериментах при $x \sim 0.049$ мы еще наблюдаем значительное положительное магнетосопротивление MR, тогда как при $x=0.093$
MR почти отсутствует, но все еще имеет положительный знак. Т. е. применяя тот же подход, оценка критической концентрации из наших экспериментов дает величину в 4 раза большую. С чем это может быть связано. Очевидно, что это должно быть связано с реальным составом и структурой образцов. В нашем случае, вследствие более высокой летучести мышьяка предполагается некоторый дефицит по этому элементу, при высоком структурном совершенстве образцов. В случае пленочных образцов, полученных в [49] термическим испарением предварительно синтезированных кристаллов, вследствие той же причины (сравнить с [48], где образцы выращиваются методом МЛЭ и обнаруживается образование второй фазы), должны получаться образцы с неоднородным распределением мышьяка по толщине образца, дефицитом кадмия в глубоко лежащих слоях и значительным числом дефектов кристаллической структуры, которые возникают при напылении образцов в режиме, далеком от режима эпитаксиального роста по механизму Франкаван дер Мерве. На наличие в образцах дефектов, связанных с наличием большого количества разорванных связей, указывает очень высокая концентрация носителей тока в пленочных образцах „чистого“ соединения, чем в случае массивных образцов, полученных традиционными методами синтеза и роста $-2.87 \cdot 10^{18} \mathrm{~cm}^{-3}$ в [49] против $6 \cdot 10^{17} \mathrm{~cm}^{-3}$ в [16].

Несмотря на то, что марганец имеет ионизационные потенциалы и сродство к электрону меньшие, чем кадмий, введение в пленочные образцы марганца [49], приводит к заметному уменьшению концентрации электронов и их подвижности, приводя к значительному росту сопротивления в сравнении с образцом без примеси марганца. В нашем эксперименте также наблюдается рост сопротивления с увеличением концентрации примеси марганца. Это указывает на реконструкцию зонной структуры и переход от дираковского полуметалла к обычному полуметаллу с параболическим законом дисперсии. Однако это происходит при более высоких концентрациях примеси марганца, вследствие более высокой когерентности кристаллической структуры, распространяющей топологическую защищенность электронных дираковских состояний на бо́льшие расстояния и, как следствие, режим слабой антилокализации до больших концентраций примесей.

\section{4. Заключение}

При легировании 3D-топологического полуметалла $\alpha-\mathrm{Cd}_{3} \mathrm{As}_{2}$ ионами марганца установлено, что легирование не меняет знак MR. MR остается также положительным и многократно увеличивается с понижением температуры. При высоких концентрациях примеси марганца происходит критическое уменьшение подвижности электронов проводимости и разрушение топологического состояния, о чем свидетельствует подавление магнетосопротивления. Критическая концентрация примеси 
марганца для образцов с совершенной кристаллической структурой и небольшим дефицитом по мышьяку значительно выше таковой для образцов с большим числом дефектов и избытком мышьяка. Ион $\mathrm{Mn}^{2+}$ размещается преимущественно в позициях замещения, и до определенных концентраций в позициях внедрения. Причем в позициях замещения полностью отсутствует плотность валентных $s$-электронов и $g$-фактор ионов $\mathrm{Mn}^{2+}$ в точности равен $g$-фактору свободного электрона. В позициях внедрения имеется присутствие поляризованных $s$-электронов, характер поляризации которых определяется конкуренцией диамагнитного вклада электронов матрицы и образующихся в ней при увеличении концентрации марганца ферромагнитных кластеров MnAs. Ион $\mathrm{Mn}^{2+}$ образует короткоживущее связанное состояние с электронами проводимости - полярон, в котором обменное взаимодействие $\mathrm{Mn}^{2+}$ с электроном проводимости имеет антиферромагнитный характер и приводит к уменьшению наблюдаемого статического магнитного момента на ионе $\mathrm{Mn}^{2+}$. При высоких концентрациях марганца, $x \sim 0.1$, когда подвижность свободных носителей тока сильно уменьшена и их $g$-фактор равен $g$-фактору свободного электрона, происходит перестройка зонной структуры со структуры дираковского полуметалла на структуру обычного полуметалла и выход из режима слабой антилокализации носителей тока. Этот переход, будучи связан с положением уровня Ферми и зависимостью химического потенциала от магнитного поля совместно с влиянием поляризованных зонных состояний ферромагнитных кластеров MnAs, обусловливает изменение полевой зависимости контактного потенциала.

\section{Благодарности}

Авторы благодарят профессора В. Мошняга (Университет Георга-Августа Гёттинген) за помощь в измерении магнитной восприимчивости.

\section{Финансирование работы}

Работа выполнена в рамках государственных заданий.

\section{Конфликт интересов}

Авторы заявляют об отсутствии конфликта интересов.

\section{Список литературы}

[1] К.С. Журавлев, Т.С. Шамирзаев, Н.А. Якушева. ФТП 32 , 791 (1998)

[2] C. Mocuta, D. Bonamy, S. Stanescu, S. El Moussaoui, A. Barbier, F. Montaigne, F. Maccherozzi, E. Bauer, R. Belkhou. Sci. Rep. 7, 16970 (2017).

[3] Younghun Hwang, Jeongyong Choi, Dang Duc Dung, Yooleemi Shin, Sunglae Cho. J. Appl. Phys. 109, 063914 (2011).
[4] S.M. Young, S. Zaheer, J.C.Y. Theo, C.L. Kane, E.J. Mele. Phys. Rev. Lett. 108, 140405 (2012).

[5] S. Borisenko, Q. Gibson, D. Evtushinsky, V. Zabolotnyy, B. Büchner, R.J. Cava. Phys. Rev. Lett. 113, 027603 (2014).

[6] C.J.M. Denissen, H. Nishihara, J.C. van Gool, W.J.M. de Jonge. Phys. Rev. 33, 7637 (1986).

[7] E.K. Arushanov. Prog. Cryst. Growth Charact. 25, 131 (1992).

[8] I. Crassee, R. Sankar, W.-L. Lee, A. Akrap, M. Orlita. Phys. Rev. Mater. 2, 120302 (2018).

[9] С.Ф. Маренкин, В.М. Трухан, И.В. Федорченко, С.В. Труханов, Т.В. Шёлковая. ЖНХ 59, 511 (2014).

[10] S.N. Jammalamadaka, S. Kuntz, O. Berg, W. Kittler, U.M. Kannan, J.A. Chelvane, C. Sürgers. Sci. Rep. 5, 13621 (2015).

[11] M. Goyal, H. Kim, T. Schumann, T. Schumann, L. Galletti, A.A. Burkov, S. Stemmer. Phys. Rev. Mater. 3, 064204 (2019).

[12] N. Marcano, S. Sangiao, M. Plaza, L. Pérez, A. Fernández Pacheco, R. Córdoba, M.C. Sánchez, L. Morellón, M.R. Ibarra, J.M. De Teresa. Appl. Phys. Lett. 96, 082110 (2010).

[13] B.L. Altshuler, A.G. Aronov, B.Z. Spivak. JETP Lett. 33, 94 (1981).

[14] S.V. Iordanskii. JETP Lett 60, 206 (1994).

[15] W.E. Liu, E.M. Hankiewicz, D. Culcer. arXiv:1708.04930v1 [cond-mat.mes-hall] 15 Aug (2017).

[14] P.W. Anderson. Phys. Rev. 124, 41 (1961).

[15] J. Kondo. Progr. Theor. Phys. 32, 37 (1964).

[16] Ю.В. Горюнов, А.Н. Натепров. ФТТ 60, 69 (2018).

[17] Ю.В. Горюнов, А.Н. Натепров. ФТТ 62, 78 (2020).

[18] M.M. Vazifeh, M. Franz. Phys. Rev. Lett. 111, 027201 (2013).

[19] M.A. Ruderman, C. Kittel. Phys. Rev. 96, 99 (1954) .

[20] N. Bloembergen, T.J. Rowland. Phys. Rev. 97, 1679 (1955).

[21] И.Я. Коренблит, Е.Ф. Шендер. УФН 126, 233 (1978).

[22] Б.И. Кочелаев, Л.Р. Тагиров, М.Г. Хусаинов. ЖЭТФ 76, 578 (1979).

[23] G.G. Khaliullin, B.I. Kochelaev. Phys. Lett. A 106, 318 (1984).

[24] T.S. Altshuler, Yu.V. Goryunov, M.S. Bresler. Phys. Rev. 73, 235210 (2006).

[25] E. Kogan. Graphene 2, 8 (2013).

[26] Hao-Ran Chang, Jianhui Zhou, Shi-Xiong Wang, Wen-Yu Shan, Di Xiao. Phys. Rev. B 92, 241103(R) (2015).

[27] Jin-Hua Sun, Dong-Hui Xu, Fu-Chun Zhang, Yi Zhou. Phys. Rev. B 92, 195124 (2015).

[28] A.N. Holden, C. Kittel, F.R. Merritt, W.A. Yager. Phys. Rev. Lett. 77, 147 (1949).

[29] С.Ф. Маренкин, А.Н. Аронов, И.В. Федорченко, А.Л. Желудкевич, А.В. Хорошилов, М.Г. Васильев, В.В. Козлов. Неорган. материалы 54, 913 (2018).

[30] В.Б. Лазарев, Н.П. Лужная, С.Ф. Маренкин. Неорган. химия 17, 3082 (1972).

[31] К.К. Палкина, В.Г. Кузнецов, В.Б. Лазарев. Неорган. химия 20, 226 (1975).

[32] C.H. Townes, J. Turkevich. Phys. Rev. Lett. 77, 148 (1949).

[33] А. Абрагам, Б. Блини. Электронный парамагнитный резонанс переходных ионов. Мир, Москва( 1973). Гл. 17. [A. Abragam, B. Bleaney. Electron Paramagnetic Resonance of Transition Ions. Oxford: Clarendon Press (1970). Ch. 17.]

[34] С.А. Альтшулер, Б.М. Козырев. Электронный парамагнитный резонанс соединений элементов промежуточных групп. Наука, М. (1972). С. 172-175.

[35] G.A. Govor. Phys. Status Solidi A 91, K59 (1985).

[36] L. Pytlik, A. Zięba. J. Magn.Magn.Mater 51, 199 (1985). 
[37] K. Maki, T. Kaneko, H. Hiroyoshi, K. Kamigaki. J. Magn. Magn. Mater 177-181, 1361 (1998).

[38] L. Däweritza, L. Wan, B. Jenichen, C. Herrmann, J. Mohanty, A. Trampert, K.H. Ploog. J. Appl. Phys. 96, 5056 (2004).

[39] A de Campos, M.A. Mota, S. Gama, A.A. Coelho, B.D. White, M.S. da Luz, J.J. Neumeier. J. Crystal Growth 333, 54, (2011).

[40] В.И. Митюк, Н.Ю. Панкратов, Г.А. Говор, С.А. Никитин, А.И. Смаржевская. ФТТ 54, 1865 (2012).

[41] N.D. Zhigadlo. J. Cryst. Growth 480, 148 (2017).

[42] C.J.M. Denissen. Analysis of the magnetic properties of semimagnetic semiconductors: an experimental study. Technische Universiteit Eindhoven. Eindhoven (1986). DOI: 10.6100/IR251727

[43] A. Abragam. Phys. Rev. 79, 534 (1950).

[44] Л.К. Аминов, И.Н. Куркин, Б.3. Малкин. ФТТ 55, 1249 (2013).

[45] F.J. Dyson. Phys. Rev. 98, 349 (1955).

[46] Л.Д. Ландау, Е.М. Лифшиц. Статистическая физика. Наука, М. (1976). Ч. 1. Гл. XIV. С. 586.

[47] R.K. Wangsness. Phys. Rev. 91, 1085 (1953).

[48] H. Wang, J. Ma, Q. Wei, J. Zhao. J. Semicond. 41, 072903 (2020).

[49] А.Б. Мехия, А.А. Казаков, Л.Н. Овешников, А.Б. Давыдов, А.И. Риль, С.Ф. Маренкин, Б.А. Аронзон. ФТП 53, 1479 (2019).

[50] Л.А. Сайпулаева, Ш.Б. Абдулвагидов, М.М. Гаджиалиев, А.Г. Алибеков, Н.В. Мельникова, Е.А. Степанова, Д.О. Аликин, В.С. Захвалинский, А.И. Риль, С.Ф. Маренкин, 3.Ш. Пирмагомедов. Физ. техн. высок. давл. 29, 4, 48 (2019).

[51] Н.П. Гражданкина. УФН 96, 2, 291 (1968).

[52] M.F. Hagedorn, W. Jeitschko. J. Solid Status Chem. 113 (2), 257 (1994).

[53] M.H. Möller, W. Jeitschko. Z. Kristallograph. - Cryst. Mater. 204, 1-2, 77 (1993).

[54] Н.Т. Гладких, А.П. Крышталь, С.И. Богатыренко. ЖТФ 80, 111 (2010).

Редактор К.В. Емцев 